\title{
Integration of bio-responsive silver in 1D photonic crystals: towards the colorimetric detection of bacteria
}

\author{
Giuseppe M. Paternò, (D *a Liliana Moscardi, ${ }^{\text {ab }}$ Stefano Donini, ${ }^{a}$

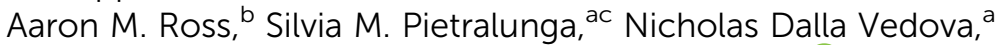 \\ Simone Normani, ${ }^{a}$ Ilka Kriegel, ${ }^{d}$ Guglielmo Lanzani (D) ab \\ and Francesco Scotognella (iD) ab
}

Received 25th February 2020, Accepted 6th March 2020

DOI: $10.1039 /$ dOfd00026d

The colour purity and versatility of fabrication of one-dimensional photonic crystals (1D $\mathrm{PhCs}$ ) make them ideal candidates for colorimetric sensing of a variety of analytes. For instance, the detection of bacterial contaminants in food via colorimetric sensors can be highly appealing, as most of the existing detection techniques are in general timeconsuming and the read-out requires specialised personnel. Here, we present a colorimetric sensor based on hybrid plasmonic/photonic 1D crystals. We demonstrate that the modification of the silver plasmon resonance brought about by the effective silver/bacterium interaction can be translated into the visible spectral region, producing a change in the structural colour. In addition, we observe a superior colorimetric sensitivity against the Gram negative Escherichia coli compared to the Gram positive Micrococcus luteus, a result that we attribute to the more efficient electrostatic interaction and cellular adhesion between the silver surface and the Gram-negative bacteria outer membrane. This approach demonstrates that in principle an easy colorimetric detection of bacterial contaminants can be achieved through the use of bio-responsive plasmonic materials, such as silver, whose selective electrostatic interaction with bacterial cell wall is well-known and occurs without the need of chemical functionalisation.

\section{Introduction}

Photonic crystals (PhCs) exhibit highly pure structural colours that can be engineered finely over the whole visible spectrum, thus offering an easy and

${ }^{a}$ Center for Nano Science and Technology, Istituto Italiano di Tecnologia (IIT), Via Pascoli 10, Milano, 20133, Italy. E-mail: Giuseppe.Paterno@iit.it

${ }^{b}$ Physics Department, Politecnico di Milano, Piazza L. da Vinci 32, 20133 Milano, Italy

'Institute for Photonics and Nanotechnologies (IFN), Consiglio Nazionale delle Ricerche (CNR), Piazza L. da Vinci 32, 20133 Milano, Italy

${ }^{d}$ Department of Nanochemistry, Istituto Italiano di Tecnologia (IIT), via Morego, 30, 16163 Genova, Italy 
recognizable colorimetric read-out while operating in a label-free fashion. ${ }^{1-3}$ These features can be appealing for the detection of contaminants and pathogenic bacteria in food and water, as the existing detection approaches rely on the use of expensive equipment and specialised personnel due to the complexity of the read-out., ${ }^{4,5}$

One-dimensional PhCs, in which the structural colour arises from the alternation of layers with high/low refractive index, have been utilised widely as versatile detection platforms ${ }^{6-9}$ owing to their well-defined optical features, to their relatively easy fabrication and compatibility with a variety of stimuliresponsive functionalities. ${ }^{10}$ In these latter regards, 1D PhCs responsiveness can be enhanced greatly by integrating a number of building blocks, including mesoporous structures, ${ }^{11-16}$ dielectric nanoparticles, ${ }^{17-20}$ polymer multilayers, ${ }^{\mathbf{8}, 21-23}$ and specific chemical functionalities able to interact with a number of pollutants and contaminants. ${ }^{24,25}$ On the other hand, detection of whole bacterial cells can be more difficult than molecular analytes, mostly due to the impossibility for micron-sized bacterial cells to percolate throughout the full multi-layered structure. In these regards, Miller and co-workers have proposed a 1D PhC resonator embedding an emissive defect layer between two 1D reflectors. By functionalizing the central layer with an organic receptor that specifically interacts with Gram negative bacterial membrane, they observed a red-shift $(4 \mathrm{~nm})$ of the photoluminescence upon exposure to Escherichia coli $(E$. coli $){ }^{26}$ These interesting studies demonstrate that 1D PhCs can be used successfully for sensing bacteria and complex contaminants, although a higher spectral shift is required for visual colorimetric identification of the analytes that can be recognized even by unskilled operators.

An alternative approach to confer responsivity to 1D PhCs consists of the integration of electro/phototunable plasmonic materials in the photonic structure. ${ }^{27-30}$ These systems, which include noble metals and doped metal oxides, besides being very sensitive to the surrounding dielectric constant, can also respond strongly to changes in their carrier density upon application of external stimuli, such as photo/electro doping. This translates into the active manipulation of their dielectric functions and, hence, of the refractive index contrast that ultimately governs the photonic band gap and the structural coloration. We have demonstrated recently that such an effect can be exploited to fabricate electrooptical switches based on the photonic reflection shift upon photo/electro doping of indium tin oxide (ITO) nanoparticles in $\mathrm{SiO}_{2} /$ ITO and $\mathrm{TiO}_{2} /$ ITO photonic crystals ${ }^{28,29}$ and electro doping of silver nanoparticles in $\mathrm{TiO}_{2} / \mathrm{Ag}$ crystals. ${ }^{27}$ It is worth mentioning that this is in-fact a physical functionalisation process that presents some advantages over chemically modified PhCs, namely enhanced environmental stability due to the reduced reactivity of the constituent elements and scalability (i.e. by using radio-frequency sputtering deposition ${ }^{31}$ ). Moreover, bio-sensing capability can be achieved for those analytes that can modify selectively the charge carrier distribution and the dielectric environment of plasmonic materials. Within this context, silver is widely recognised and employed as a bio-responsive plasmonic material. ${ }^{32-34}$ The bactericidal activity of silver seems to be linked to the strong electrostatic adhesion between the negatively charged bacterial outer membrane and the positively charged silver ions, followed by efficient $\mathrm{Ag}^{+}$uptake inside the bacterial cells. ${ }^{35-37}$ We have preliminarily determined that this might lead to a modification of $\mathrm{Ag}$ charge carrier 
density and shift of the plasmon resonance upon E. coli/Ag interaction. ${ }^{38}$ In addition, interestingly, by integrating the plasmonic functionality in the $1 \mathrm{D}$ PhC, we have observed that such a plasmon modulation brought about by the bacterium can be translated into a photonic read-out, although a precise colorimetric response was not achieved. ${ }^{38}$

Here, we build on those previous studies and report the colorimetric detection of the Gram negative E. coli. In addition, we extend our investigation to the Gram positive Micrococcus luteus (M. luteus). We attribute the less obvious photonic response to this latter bacterium strain to the different outer membrane/silver interaction in Gram positive bacteria.

\section{Experimental section}

\section{Photonic crystals fabrication}

1D PhCs were fabricated by sequential deposition of $\mathrm{SiO}_{2}$ and $\mathrm{TiO}_{2}$ layers via spincasting from their colloidal aqueous dispersions following the procedure employed in past experiments. ${ }^{28,29}$ Firstly, we suspended $\mathrm{TiO}_{2}$ (Gentech Nanomaterials, average size $5 \mathrm{~nm}$ ) and $\mathrm{SiO}_{2}$ nanoparticles (Sigma Aldrich LUDOX SM30 , average size $8 \mathrm{~nm}$ ) in MilliQ distilled water to obtain a concentration of $10 \mathrm{wt}$ $\%$. The dispersions were then tip-sonicated for $10 \mathrm{~min}$ and filtered with a $0.45 \mu \mathrm{m}$ PVDF filter. The glass substrates $(2 \times 2 \mathrm{~cm})$ were previously cleaned by means of ultra-sonication in isopropanol (10 $\mathrm{min}$ ) and acetone $(10 \mathrm{~min})$, and then oxygen plasma treated (Colibri Gambetti, $10 \mathrm{~min}$ ) to increase wettability. During the fabrication of the PhCs, the dispersions were continuously kept in sonication at $45{ }^{\circ} \mathrm{C}$ to maintain homogeneity during the whole fabrication process. Spincoating (Laurell WS-400-6NNP-Lite) was carried out with a speed of $2000 \mathrm{rpm}$. After each deposition, samples were annealed on a hot-plate for $20 \mathrm{~min}$ at $350^{\circ} \mathrm{C}$. The thickness of each layer was around $380 \mathrm{~nm}$ for $\mathrm{SiO}_{2}$ and $100 \mathrm{~nm}$ for $\mathrm{TiO}_{2}$. Finally, we deposited an $8 \mathrm{~nm}$-thick silver layer on top of the photonic structure (or glass substrate only) via thermal evaporation (MBRAUN metal evaporator).

\section{Bacterial culture}

A single colony from the Escherichia coli ATCC25922 strain was inoculated in Luria-Bertani (LB) broth and incubated overnight at $37{ }^{\circ} \mathrm{C}$ with shaking at $200 \mathrm{rpm}$ until a stationary phase was reached. Then, the bacterial suspension turbidity (expressed as optical density at $600 \mathrm{~nm}$; O.D. ${ }_{600}$ ) was diluted to O.D. ${ }_{600} \sim$ 0.5 (corresponding to $1.12 \times 10^{7}$ colony-forming units (cfu) per $\mathrm{mL}$ ) in LB broth (no antibiotic). The suspension $(500 \mu \mathrm{L})$ was spread over an LB agar plate. The Ag/ PhCs were placed at the center of the Petri dish with the top silver layer facing the contaminated surface (or LB only for the control experiment) and incubated for $24 \mathrm{~h}$ at $37^{\circ} \mathrm{C}$. Furthermore, the same experimental procedure was adopted for the Gram positive Micrococcus luteus ATCC 9341. In this case, we used tryptic soy agar (TSA) culture medium.

\section{Optical characterization}

The optical transmittance was recorded using a fiber-coupled spectrometer (Avantes, AvaSpec-HS2048XL-EVO) equipped with a deuterium-halogen lamp (AvaLight-D(H)-S). Transmittance spectra were corrected for blank (bare glass 
substrate) and dark signal. We took a minimum of three measurements on each sample usually in the central region. We could not observe significant spectral differences among measurements on the same sample. Data were taken over two sets of measurements (three samples per measurement).

\section{Scanning electron microscopy}

Images were taken using a Tescan MIRA3 High-RES SEM. Secondary electron imaging was performed at a voltage of $5 \mathrm{kV}$ and with currents in the pA range.

Silver layers were deposited on top of silicon p-doped substrates and covered with carbon paste to improve conductivity.

\section{Results and discussion}

Firstly, we analyse the morphological and optical behaviour of silver films on top of non-photonic substrates (silicon for SEM characterisation and glass for absorption measurements) upon contamination with E. coli and M. luteus. Scanning electron microscopy images of pristine and $E$. coli-contaminated silver films are reported in Fig. 1a and b. Thermal evaporation of a thin layer of silver leads to a discontinuous and nanostructured morphology, with nanoislands

a

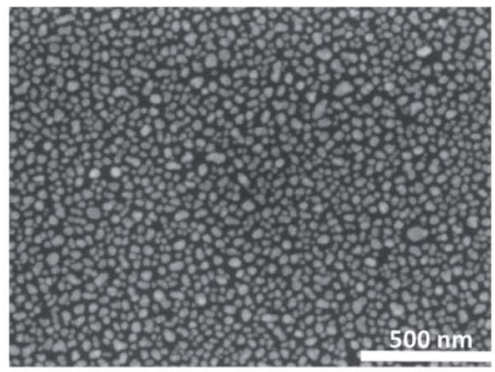

b

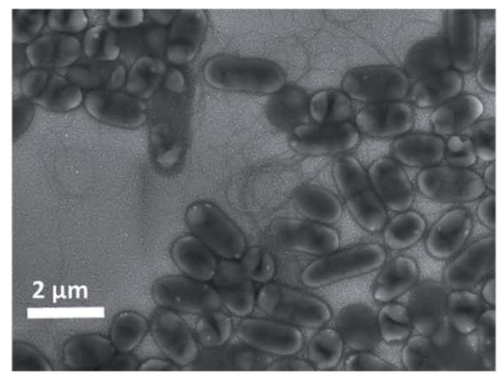

d
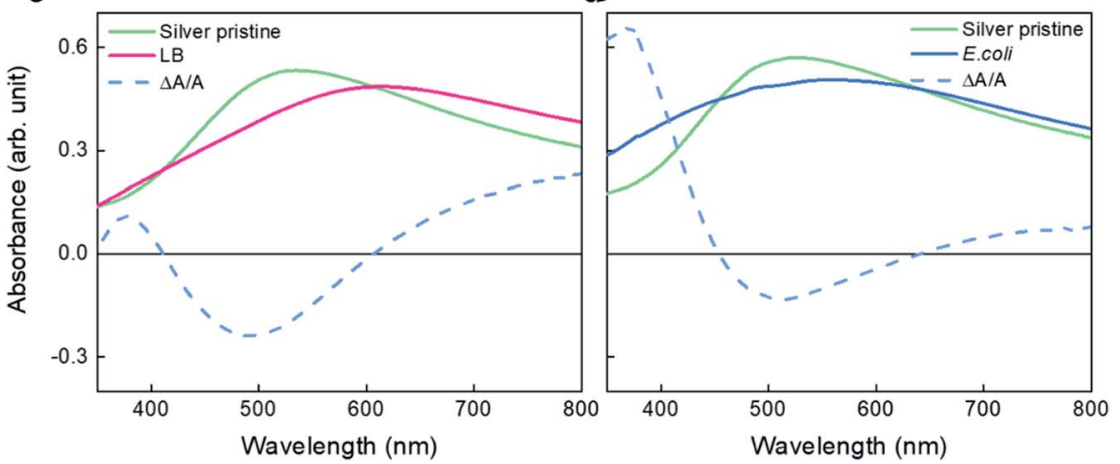

Fig. 1 SEM images of the silver layer ( $8 \mathrm{~nm}$ thickness on silicon substrates) before (a) and after (b) exposure to E. coli. (c) Silver optical absorption $(8 \mathrm{~nm}$ thickness on glass substrates) before (silver pristine) and after exposure to the LB culture medium and (d) to $E$. coli. The blue dotted lines represent the differential absorption expressed as $\left(\right.$ Abs $_{E .}$ coli Abs pristine)/Abs pristine. 
exhibiting a broad distribution of sizes (diameter 20-50 nm). The silver layer was then exposed to $E$. coli $\left(10^{7}\right.$ colony forming units, $\left.\mathrm{cfu}\right)$ in an agar plate. E. coli cells present the usual rod morphology, although an important fraction of cells $(\approx 50 \%)$ seem to undergo a change from rod to circular morphology, possibly caused by lack of separation of the daughter cells during the division cycle in such an unfavourable environment. ${ }^{39}$ The bright regions that are mostly distributed in the cell outer membrane can be attributed to the silver ions taken up by the bacterial cells, which is the initial step of the bactericidal process. ${ }^{39-41}$ To assess the optical transmission of the silver film upon contamination, we spread the bacterial suspension in the culture medium (Luria-Bertani broth, LB) on agar gel at one specific concentration (0.5 O.D. 600 corresponding to $1 \times 10^{7}$ colonyforming units per $\mathrm{mL}$ ) and exposed our samples to such contaminated surface or to the non-contaminated culture LB medium as a control measurement (Fig. 1c and d). Note that such a bacterial concentration is used for challenge studies on real food matrices. ${ }^{42}$ The absorption peak centred at around $520 \mathrm{~nm}$ can be related to the surface plasmon resonance, ${ }^{43}$ which is inhomogeneously broadened as a result of the wide distribution of sizes of the silver nanoislands (full width at half maximum, FWHM $=430 \mathrm{~nm}$ ). If we expose the surface to the culture medium only, we observe a clear red-shift of the plasmon absorption $(80 \mathrm{~nm})$, a result that we attribute to the increase in the effective refractive index ${ }^{38,44}$ due to the infiltration of the aqueous culture medium within the silver nanoislands. On the other hand, the marked FWHM increase (from $430 \mathrm{~nm}$ to $610 \mathrm{~nm}$ ) can be connected to the enhanced energetic disorder owing to the complex dielectric environment of the culture medium..$^{45}$ Interestingly, E. coli contamination leads to a substantial increase of the high energy wing of the plasmon absorption (350$450 \mathrm{~nm})$, an effect that can be appreciated better by plotting the differential absorption spectra $\left[\left(\mathrm{Abs}_{E \text {. coli }}-\mathrm{Abs}_{\text {pristine }}\right) / \mathrm{Abs}_{\text {pristine, }}\right.$ dotted blue lines in Fig. 1c and d]. In our previous investigation, ${ }^{38}$ we speculated that such a shift might originate from the formation of polarization charges at the silver-bacterium interface, eventually causing an overall increase in the charge carrier density. ${ }^{27,28}$ However, we cannot rule out concomitant yet important causes, such as modifications of particle size brought about by silver uptake that might determine appreciable shifts in the plasmon frequency. Further experiments are currently underway to obtain insight in these regards.

The SEM images of the Gram-positive M. luteus on the silver substrate are reported in Fig. 2a and b. This bacterium is of interest in the field of food safety, as it has been found recently in contaminated chicken. ${ }^{46}$ In this case, the typical spherical shape of Micrococci seems to be retained upon exposure to nanostructured silver. Furthermore, we could not observe strong indications of silver uptake by $M$. luteus cells as in the case of $E$. coli. Passing to the optical characterisation (Fig. 2c and d), we note that the red-shift $(80 \mathrm{~nm})$ caused by TSA is consistent with what is observed for LB agar. Conversely, contamination with $M$. luteus leads to a convolution of spectral effects, including: (i) a dramatic increase of the FWHM (from $490 \mathrm{~nm}$ to $1000 \mathrm{~nm}$ ); (ii) the usual red-shift ( $80 \mathrm{~nm}$ ) of the plasmon resonance that can be linked to the culture medium; (iii) an increase of the absorption in the UV region. These findings suggest that also the Grampositive bacterium can modify the silver plasmon resonance, even though the intricate optical read-out observed after contamination with M. luteus suggests a less sensitive interaction between the silver surface and this latter bacterium. 
a

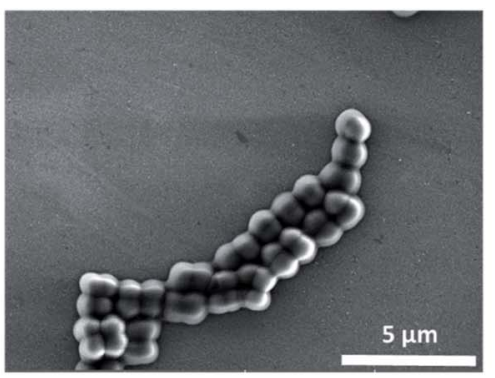

c

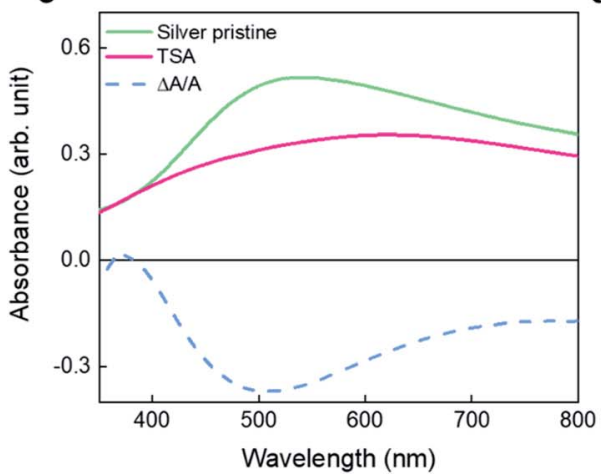

b

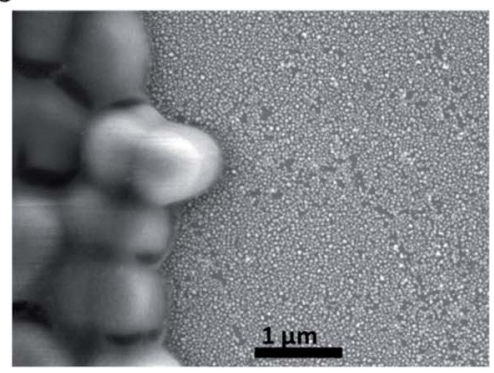

d

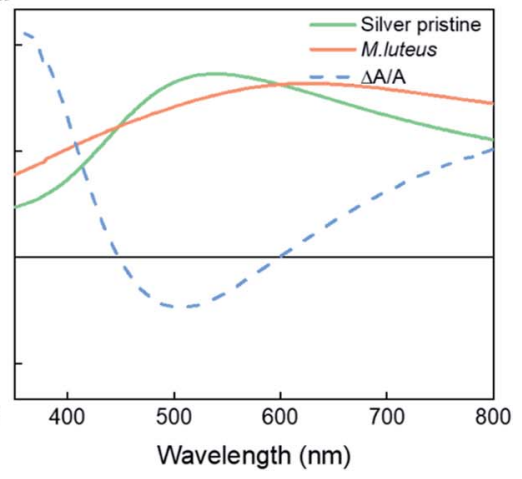

Fig. 2 SEM images of M. luteus on silver at low (a) and high magnification (b). (c) Silver optical absorption before (silver pristine) and after exposure to the TSA culture medium and (d) to M. luteus. Also in this case, the blue dotted lines represent the differential absorption expressed as $\left(\mathrm{Abs}_{M}\right.$. Luteus $\left.-\mathrm{Abs}_{\text {pristine }}\right) / \mathrm{Abs}_{\text {pristine. }}$

The different cell envelopes in Gram-negative and Gram-positive bacteria might provide a preliminary explanation of these results. In particular, Gramnegative bacteria possess an outer membrane (OM) consisting of an inner phospholipidic bilayer and an outer leaflet that is mostly composed of negatively charged lipopolysaccharides. ${ }^{47,48}$ In contrast, Gram-positive bacteria present a thick layer of peptidoglycan to the extracellular medium. ${ }^{49}$ The higher biocidal activity of silver against Gram-negative bacteria than the Gram-positive counterparts has been indeed related to the electrostatic interaction between the negatively charged functional groups in the $\mathrm{OM}$ and the $\mathrm{Ag}^{+}{ }^{+}{ }^{50}$ This, in turn, would explain the higher optical sensitivity of the plasmon response to $E$. coli than $M$. luteus.

Although the silver plasmon resonance is already responsive optically to the presence of bacteria, a more precise colorimetric read-out in terms of optical purity in a more favourable spectral region is still highly desirable. With this in mind, we integrated the bio-responsive silver layer on top of a 1D PhC consisting of alternating layers of $\mathrm{SiO}_{2}$ and $\mathrm{TiO}_{2}$ nanoparticles (see Fig. 3a for a simplified sketch). SEM cross sectional analysis (Fig. 3b) highlights the porous structure of the photonic multilayer (estimated filling factor $=0.6-0.7$ ), ${ }^{38}$ and indicates a thickness of $\approx 380 \mathrm{~nm}$ and $\approx 100 \mathrm{~nm}$ for $\mathrm{SiO}_{2}$ and $\mathrm{TiO}_{2}$ layers, respectively. In this context, the plasmonic metal can be seen as a defective layer of the photonic 
$\mathbf{a}$

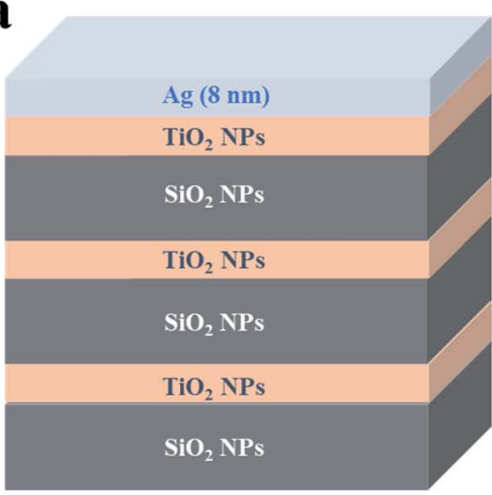

Fig. 3 (a) Sketch of the photonic crystal, consisting of a multilayer of $\mathrm{SiO}_{2}$ and $\mathrm{TiO}_{2}$ nanoparticles. In this sketch we report 3 bilayers for simplicity, while actual devices consist of 5 bilayers. (b) SEM cross section of the multilayer.

crystal that affects the optical response of the PhC via its free carrier density. ${ }^{51}$ Thus, the main goal is to modify the dielectric properties at the $\mathrm{PhC} / \mathrm{metal}$ interface and, thus, the overall optical read-out by exploiting the possible change in the silver complex dielectric function caused by the $\mathrm{Ag} / \mathrm{bacteria}$ interaction. To localise strongly the plasmonic response at the interface of the photonic structure, we selected the minimum Ag thickness attainable with our deposition apparatus.

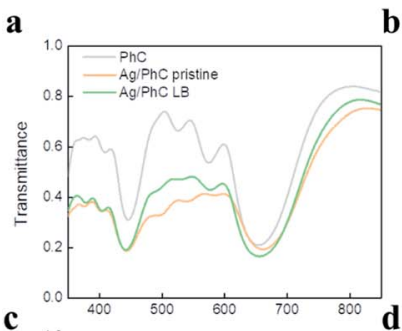

b
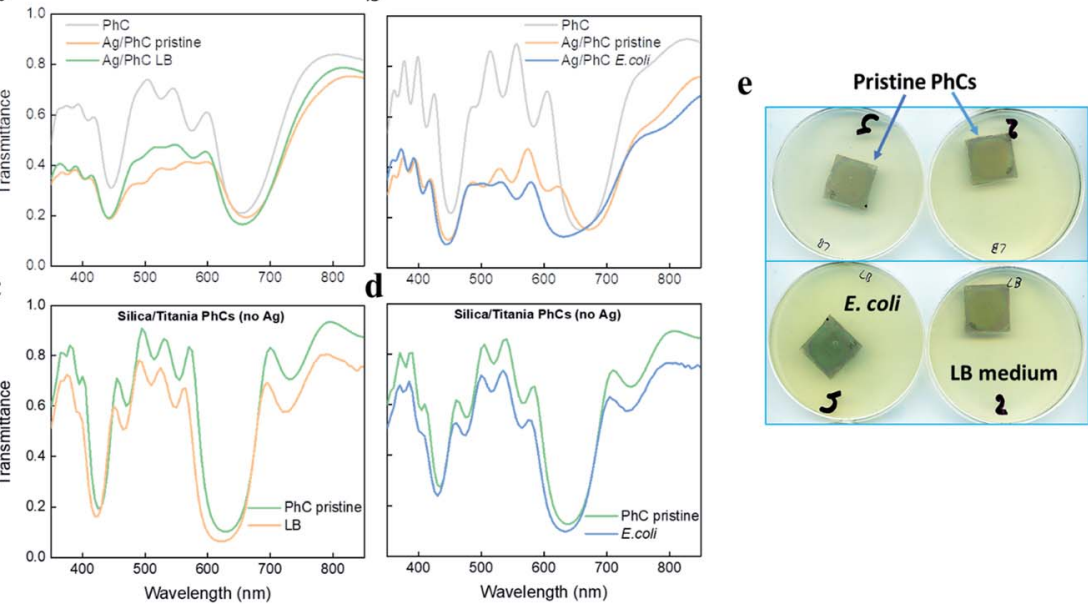

Fig. 4 (a) Transmittance spectra of the 1D PhCs before and after silver deposition, and upon exposure to the culture medium and (b) after exposure to E. coli. (c) Transmittance spectra of the 1D PhCs without silver upon exposure to the LB medium and (d) after exposure to E. coli. (e) Colorimetric change of the photonic crystals after contamination with E. coli, while exposure to LB does not cause any substantial colorimetric change. Data were averaged over two sets of measurements (three samples per measurement). For $E$. coli contaminated PhCs the average blue-shift observed was $30 \pm 10 \mathrm{~nm}$. 
The transmittance spectra (Fig. 4) show a relatively high optical quality for the fabricated devices, as highlighted by interference fringes that denote well-defined interfaces between the layers, and by the presence of the $2^{\text {nd }}$ order reflection peak at $440 \mathrm{~nm}$. Such a relatively high optical quality of these devices was achieved through optimization of the fabrication procedure, such as in terms of better dispersion of the colloidal nanoparticles attained by using tip-sonication. The thermal evaporation of the thin silver layer on top of the photonic structures leads to a reduction of the transmission due to the broad plasmon absorption, as well as a red-shift of the photonic band-gap. Interestingly, while interaction with the culture medium does not alter appreciably the photonic read-out (Fig. 4a), when the hybrid structure is interfaced with the bacterial colonies we noticed an average blue-shift of $40 \mathrm{~nm}$ of the photonic band-gap (Fig. $4 \mathrm{~b}$ ), while the average shift was $30 \pm 10 \mathrm{~nm}$. Identical experiments repeated on $1 \mathrm{D}^{\mathrm{SiO}}{ }_{2} / \mathrm{TiO}_{2}$ photonic crystals without the silver layer did not lead to any substantial shift of the photonic band-gap, indicating strongly that the metallic layer is indeed the responsive functionality in our hybrid device. Notably, the magnitude of such optical shifts allows a visual read-out of the contamination, as it can be seen in Fig. 4e. The colour seems to shift from a light to a deep green, as a result of the different spectral response of the PBG centred in the red and the $2^{\text {nd }}$ order centred in the blue, which give rise to the green colour of the crystals. In particular, while the PBG blue-shifts substantially, the second order does not change significantly, producing a deeper green coloration than in the pristine sample. A quantitative study of the visual response vs. bacteria concentration is currently underway.

To assess the analyte selectivity of our device, we thus exposed the Ag/1D PhCs to $M$. luteus following the same experimental procedure for $E$. coli contamination (Fig. 5a and b). In analogy to what was observed in E. coli contaminated silver layers, while exposure to TSA leads to a red-shift of the photonic band-gap, $M$. luteus produces a more convoluted photonic response. In particular, we notice both a red-shift that can be attributed to the culture medium, and a decreased transmittance at the blue-side of the photonic band-gap $(560-620 \mathrm{~nm})$ that instead can be related to the photonic transduction. In general, we can

a

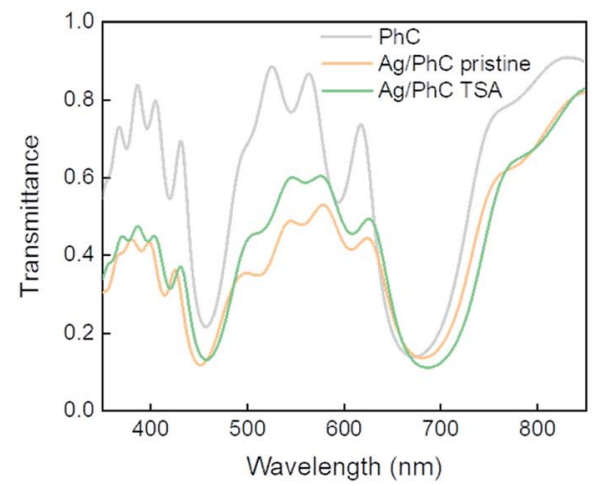

b

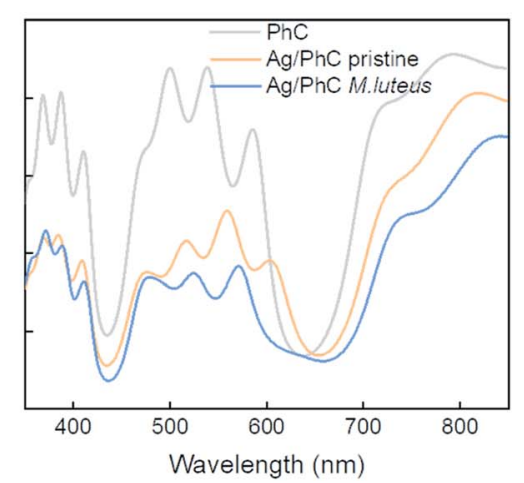

Fig. 5 (a) Transmittance spectra of the 1D PhCs before and after silver deposition, and upon exposure to TSA and (b) after exposure to M. luteus. 
preliminarily conclude that our device is more sensitive to Gram-negative bacteria than their Gram-positive counterparts.

\section{Conclusions}

In conclusion, we have reported on the colorimetric detection of bacteria contaminants by means of a hybrid plasmonic/photonic 1D crystal. The modification of the plasmon charge density and, hence, of the silver effective dielectric function caused by specific silver/bacteria interaction, such as electrostatic adhesion and silver ion uptake, might explain the clear spectral shifts of the silver plasmon resonance upon contamination. The colour purity of 1D PhCs allows for the translation of such a shift occurring in the UV region into the visible, allowing an easy colorimetric read-out of the contamination. We preliminarily found that our approach is in principle more sensitive to Gram-negative than Gram-positive bacteria, a result that we attribute to the more effective electrostatic interaction and cellular adhesion between the negatively charged outer membrane of Gramnegative bacteria and the positively charged silver ions. Additional experiments with different plasmonic bio-responsive materials and bacterial strains are currently being carried out to gain more insights into these interesting aspects.

\section{Conflicts of interest}

The authors declare financial support from the company "Centro Grafico dg S.p.A", which is collaborating with the authors for the commercialization of the products based on the findings of this work. The authors clarify that the scientific conclusions that are presented in this study are not influenced by the activity of the company.

\section{Acknowledgements}

This work has been supported by Fondazione Cariplo, grant no. 2018-0979 and grant no. 2018-0505. F. S. thanks the European Research Council (ERC) under the European Union's Horizon 2020 research and innovation programme (grant agreement No. [816313]). We thank Fabio Marangi for assisting during the SEM characterisation.

\section{References}

1 K. Szendrei-Temesi, A. Jiménez-Solano and B. V. Lotsch, Adv. Mater., 2018, 30, 1-6.

2 J. Ge and Y. Yin, Angew. Chem., Int. Ed., 2011, 50, 1492-1522.

3 R. Xiong, J. Luan, S. Kang, C. Ye, S. Singamaneni and V. V. Tsukruk, Chem. Soc. Rev., 2020, 49, 983-1031.

4 W. Meulebroeck, H. Thienpont and H. Ottevaere, IEEE Instrum. Meas. Mag., 2016, 19, 35-45.

5 W. Meulebroeck, H. Thienpont and H. Ottevaere, IEEE Instrum. Meas. Mag., 2017, 20, 46-55.

6 C. Fenzl, T. Hirsch and O. S. Wolfbeis, Angew. Chem., Int. Ed., 2014, 53, 33183335. 
7 H. Shen, Z. Wang, Y. Wu and B. Yang, RSC Adv., 2016, 6, 4505-4520.

8 P. Lova, G. Manfredi and D. Comoretto, Adv. Opt. Mater., 2018, 6, 1800730.

9 H. Inan, M. Poyraz, F. Inci, M. A. Lifson, M. Baday, B. T. Cunningham and

U. Demirci, Chem. Soc. Rev., 2017, 46, 366-388.

10 L. Nucara, F. Greco and V. Mattoli, J. Mater. Chem. C, 2015, 3, 8449-8467.

11 V. Robbiano, G. M. Paternò, A. A. La Mattina, S. G. Motti, G. Lanzani, F. Scotognella and G. Barillaro, ACS Nano, 2018, 12, 4536-4544.

12 M. C. Fuertes, F. J. López-Alcaraz, M. C. Marchi, H. E. Troiani, V. Luca, H. Míguez and G. J. A. A. Soler-Illia, Adv. Funct. Mater., 2007, 17, 1247-1254.

13 B. Auguié, M. C. Fuertes, P. C. Angelomé, N. L. Abdala, G. J. A. A. Soler Illia and A. Fainstein, ACS Photonics, 2014, 1, 775-780.

14 M. C. Fuertes, S. Colodrero, G. Lozano, A. R. González-Elipe, D. Grosso, C. Boissière, C. Sánchez, G. J. de A. A. Soler-Illia and H. Míguez, J. Phys. Chem. C, 2008, 112, 3157-3163.

15 M. C. Sansierra, J. Morrone, F. Cornacchiulo, M. C. Fuertes and P. C. Angelomé, ChemNanoMat, 2019, 5, 1289-1295.

16 V. Robbiano, G. M. Paternò, G. F. Cotella, T. Fiore, M. Dianetti, M. Scopelliti, F. Brunetti, B. Pignataro and F. Cacialli, J. Mater. Chem. C, 2018, 6, 2502-2508.

17 S. Colodrero, M. Ocaña and H. Míguez, Langmuir, 2008, 24, 4430-4434.

18 V. González-Pedro, M. E. Calvo, H. Míguez and Á. Maquieira, Biosensors and Bioelectronics: $X, 2019, \mathbf{1}, 100012$.

19 D. P. Puzzo, F. Scotognella, M. Zavelani-Rossi, M. Sebastian, A. J. Lough, I. Manners, G. Lanzani, R. Tubino and G. A. Ozin, Nano Lett., 2009, 9, 42734278.

20 F. Scotognella, D. P. Puzzo, A. Monguzzi, D. S. Wiersma, D. Maschke, R. Tubino and G. A. Ozin, Small, 2009, 5, 2048-2052.

21 P. Lova, G. Manfredi, L. Boarino, A. Comite, M. Laus, M. Patrini, F. Marabelli, C. Soci and D. Comoretto, ACS Photonics, 2015, 2, 537-543.

22 P. Lova, P. Giusto, F. Di Stasio, G. Manfredi, G. M. Paternò, D. Cortecchia, C. Soci and D. Comoretto, Nanoscale, 2019, 11, 8978-8983.

23 P. Lova, G. Manfredi, C. Bastianini, C. Mennucci, F. Buatier De Mongeot, A. Servida and D. Comoretto, ACS Appl. Mater. Interfaces, 2019, 11, 1687216880 .

24 L. D. Bonifacio, D. P. Puzzo, S. Breslau, B. M. Willey, A. McGeer and G. A. Ozin, Adv. Mater., 2010, 22, 1351-1354.

25 L. D. Bonifacio, G. A. Ozin and A. C. Arsenault, Small, 2011, 7, 3153-3157.

26 S. Chan, S. R. Horner, P. M. Fauchet and B. L. Miller, J. Am. Chem. Soc., 2001, 123, 11797-11798.

27 E. Aluicio-Sarduy, S. Callegari, D. G. F. del Valle, A. Desii, I. Kriegel and F. Scotognella, Beilstein J. Nanotechnol., 2016, 7, 1404-1410.

28 G. M. Paternò, C. Iseppon, A. D’Altri, C. Fasanotti, G. Merati, M. Randi, A. Desii, E. A. A. Pogna, D. Viola, G. Cerullo, F. Scotognella and I. Kriegel, Sci. Rep., 2018, 8, 3517.

29 G. M. Paternò, L. Moscardi, I. Kriegel, F. Scotognella and G. Lanzani, J. Photonics Energy, 2018, 8, 1.

30 S. Heo, A. Agrawal and D. J. Milliron, Adv. Funct. Mater., 2019, 29, 1904555.

31 F. Scotognella, A. Chiasera, L. Criante, E. Aluicio-Sarduy, S. Varas, S. Pelli, A. Łukowiak, G. C. Righini, R. Ramponi and M. Ferrari, Ceram. Int., 2015, 41, 8655-8659. 
32 G. Chen, Q. L. Feng, J. Wu, G. Q. Chen, F. Z. Cui, T. N. Kim and J. O. Kim, J. Biomed. Mater. Res., 2000, 52, 662-668.

33 S. D. Ponja, S. K. Sehmi, E. Allan, A. J. Macrobert, I. P. Parkin and C. J. Carmalt, ACS Appl. Mater. Interfaces, 2015, 7, 28616-28623.

34 G. Franci, A. Falanga, S. Galdiero, L. Palomba, M. Rai, G. Morelli and M. Galdiero, Molecules, 2015, 20, 8856-8874.

35 O. M. Bondarenko, M. Sihtmäe, J. Kuzmičiova, L. Ragelienė, A. Kahru and R. Daugelavičius, Int. J. Nanomed., 2018, 13, 6779-6790.

36 Z. Xiu, Q. Zhang, H. L. Puppala, V. L. Colvin and P. J. J. Alvarez, Nano Lett., 2012, 12, 4271-4275.

37 B. Le Ouay and F. Stellacci, Nano Today, 2015, 10, 339-354.

38 G. M. Paternò, L. Moscardi, S. Donini, D. Ariodanti, I. Kriegel, M. Zani, E. Parisini, F. Scotognella and G. Lanzani, J. Phys. Chem. Lett., 2019, 10, 4980-4986.

39 C. E. Peña-González, E. Pedziwiatr-Werbicka, T. Martín-Pérez, E. M. Szewczyk, J. L. Copa-Patiño, J. Soliveri, J. Pérez-Serrano, R. Gómez, M. Bryszewska, J. Sánchez-Nieves and F. J. de la Mata, Int. J. Pharm., 2017, 528, 55-61.

40 N. Durán, M. Durán, M. B. de Jesus, A. B. Seabra, W. J. Fávaro and G. Nakazato, Nanomedicine, 2016, 12, 789-799.

41 J. A. Lemire, J. J. Harrison and R. J. Turner, Nat. Rev. Microbiol., 2013, 11, 371384.

42 A. Echeverry, J. Chance Brooks, M. F. Miller, J. A. Collins, G. H. Loneragan and M. M. Brashears, J. Food Prot., 2010, 73, 2169-2179.

43 M. A. Cazalilla, J. S. Dolado, A. Rubio and P. M. Echenique, Phys. Rev. B: Condens. Matter Mater. Phys., 2000, 61, 8033-8042.

44 J. J. Mock, D. R. Smith and S. Schultz, Nano Lett., 2003, 3, 485-491.

45 G. Bertani, Studies on lysogenesis. I. The mode of phage liberation by lysogenic Escherichia coli., 1951, vol. 62.

46 Food poisoning risk of handling packs of supermarket chicken - Telegraph, https://www.telegraph.co.uk/news/health/news/8318515/Food-poisoning-riskof-handling-packs-of-supermarket-chicken.html, accessed 8 August 2019.

47 A. Boags, P. C. Hsu, F. Samsudin, P. J. Bond and S. Khalid, J. Phys. Chem. Lett., 2017, 8, 2513-2518.

48 L. A. Clifton, M. W. A. Skoda, E. L. Daulton, A. V. Hughes, A. P. Le Brun, J. H. Lakey and S. A. Holt, J. R. Soc., Interface, 2013, 10, 20130810.

49 A. Abbaszadegan, Y. Ghahramani, A. Gholami, B. Hemmateenejad, S. Dorostkar, M. Nabavizadeh and H. Sharghi, J. Nanomater., 2015, 2015, 1-8.

50 S. Shrivastava, T. Bera, A. Roy, G. Singh, P. Ramachandrarao and D. Dash, Nanotechnology, 2007, 18, 225103.

51 P. Drude, Ann. Phys., 1902, 312, 687-692. 